\title{
Entre Argentina e Italia pasando por Yugoslavia: Cruzando el río en bicicleta de Ana Cecilia Prenz Kopušar
}

Palabras clave: Argentina, Italia, Yugoslavia, auto ficción, memoria, identidad DOI: 10.4312/ars.11.2.220-231

\section{El relato}

Cruzando el río en bicicleta es un libro entre narrativa y biografía que cuenta los acontecimientos intricados de la vida de una adolescente. La infancia y la juventud transcurridos entre Belgrado, La Plata y Trieste la llevan a una formación internacional en la que la protagonista entra en contacto con sistemas sociales y políticos opuestos: la Yugoslavia de Tito con su fuerte sentido de pertenencia, la dictadura argentina de la que la familia escapa, la extraña libertad de Trieste. La mujer recuerda su crecimiento entre lenguas, culturas, lugares de tres países distintos y lo cuenta con el desenfado de una joven curiosa, sedienta de vida, que se asoma al mundo.

Profesora de la Universidad de Trieste, nacida en Belgrado (Serbia), de padres argentinos, Ana Cecilia Prenz Kopušar pasa su infancia y primera juventud entre Argentina, Yugoslavia e Italia. Estudia en Roma, en la Universidad de La Sapienza, donde se licencia en Disciplinas del Espectáculo y se doctora en la Universidad de La Plata. El elemento intercultural en teatro y en literatura ha sido el tema constante de su trabajo de investigadora; algunos frutos de esta labor son los ensayos «K. S. Stanislawskij y el Teatro Argentino» (1999) y «Contigüidades culturales en las piezas romanas de Bartolomé Torres Naharro» (2008). Se ocupa también de la literatura sefardí en Bosnia, en particular de la dramaturga Laura Papo Bohoreta, de la que ha publicado el primer y el segundo tomo de sus manuscritos $(2015,2016)$. Además de investigadora, es también traductora del/al español, italiano, serbocroata y esloveno; ejemplos de esta actividad son Vísteme con un beso (2012) de la escritora y actriz eslovena Saša Pavček y Sono due quelli che danzano - Ples $v$ dvoje (2013) del poeta argentino José María Pallaoro. Vive entre Trieste y Kamna Gorica (Eslovenia), donde dirige el centro intercultural «La casa de Kamna», lugar de encuentro y reflexión sobre América Latina. A todos estos intereses se añade también el de escritora y Cruzando el río en bicicleta - Attraversando il fiume in bicicletta es su primera obra en este ámbito. 
El tomo se publicó en Buenos Aires en 2015 (con una primera edición en 2013) y la misma Cecilia Prenz lo tradujo en italiano en 2016, cambiando algunas partes. La traducción serbia, en cambio, se encuentra en fase de elaboración y la autora la está realizando con Aleksandar Lazić, entre otras cosas el personaje del libro llamado Saša. La revisión de la traducción es de Marija Mitrović.

El libro pertenece a un género lábil, acaso una novela biográfica, o más bien una narración que entra en lo que hoy se define como autoficción, donde el yo narrador se acerca y se aleja del referente. En la constante búsqueda de recuerdos de la infancia y adolescencia, la tensión entre la figura del yo y el objeto/representado varía. Así Prenz ensaya una serie interesante de estrategias que desestabilizan los límites entre ficción y realidad. Es la misma autora quien afirma:

En algún momento he tomado en consideración el término autoficción. Muchos son los elementos autobiográficos que construyen el texto. Sin embargo, estos son elementos funcionales para contar otra historia, que es una historia más amplia, de personajes y de países (en guerra, bajo dictadura y en democracia), de sus relaciones, de lo que fue o habría podido ser, de sus complejidades y alegrías; y todo ello visto a través de los ojos de esa adolescente que ofrece su visión de la realidad, que cuenta su verdad. Muchos son los episodios que remiten a planteos propios de nuestra contemporaneidad. No nos encontramos ante esa distancia histórica que coloca al lector frente a hechos lejanos en el tiempo y en el espacio, o ajenos temáticamente. El libro trata argumentos, como la dictadura argentina y la guerra yugoslava, cuyas secuelas están aún presentes en los respectivos contextos sociales porque políticamente no fueron resueltas. En este sentido, varios fragmentos del texto podrían ser leídos e interpretados a partir de distintas perspectivas y con distintas intencionalidades... (Prenz, 2017, en prensa)

Gracias a la acción del recuerdo, el trabajo de la memoria, como explica Laura Scarano, «desarticula las cronologías, mezcla episodios, desplaza lugares, crea un personaje diferente sin disolver la paradoja que nos remite difusamente a la figura del autor y autoriza la autoficción» $(2007,91)$. De esta forma se alimenta un subgénero híbrido, caracterizado por la duda entre la realidad y la ficción donde le compete al lector definir estos límites. El propio Puertas Moya, estudiando la transformación de una vida en fantasía con un/a protagonista en parte inventado/a, señala que la autoficción «ha puesto al descubierto el carácter ficcional del yo autobiográfico, su inexistencia real fuera de los márgenes y máscaras que le presta la literatura» $(2005,325)$.

Cecilia Prenz reconoce el carácter mixto de su libro y afirma: «Creo que están presentes los dos elementos. Sin los elementos autobiográficos que construyen la 
historia de la adolescente y joven protagonista de la nouvelle no habría posibilidad de narración» (Crolla, 2016).

Cruzando el río en bicicleta es un texto relativamente breve como número de páginas pero muy denso por la amplitud de las experiencias relatadas, con una primera persona que recuerda a través de diecisiete rápidos capítulos sus años de infancia y adolescencia pasados entre Belgrado -ciudad amada y protagonista de parte de la historia-, Argentina, más precisamente La Plata y Ensenada, tema presente en varios capítulos («Ford Falcon», «La lengua», «Buenos Aires», «El río, los sueños», «La bandera argentina», «Tomás») y Trieste, donde la joven descubre la belleza.

La narración no sigue un orden cronológico ni geográfico, sino que empieza con su adolescencia en Belgrado para continuar en Argentina y enseguida volver a Yugoslavia y continuar con Trieste para terminar en Sarajevo. La alternancia aparentemente desordenada presenta, sin embargo, una articulación armónica entre lugares y tiempos distintos, la narración avanza con fluidez y acompaña la formación de esta joven mujer entusiasta y generosa. El período que abarca la historia relatada se refiere a los años de la última dictadura en Argentina, caracterizada por una violencia cada vez más evidente que obliga la familia Prenz a dejar el país para refugiarse en la ciudad donde los padres ya habían transcurrido algunos años y donde había nacido su primera hija. En Belgrado viven cuatro años para trasladarse - sin perder nunca el contacto con la capital serbia- a Trieste después de la muerte de Tito, cuando empieza la crisis de la región que dará paso a la desintegración del país en las actuales seis repúblicas.

Junto al tema del recuerdo, sobre el que se funda la narración, se encuentra el de la identidad, que remite a un imperativo del sujeto y a una exigencia universal. Entre lugares, olores y sabores, todos muy presentes, se percibe un yo esquivo con una identidad incierta que alterna una atracción por las distintas geografías que componen su existencia. El recuerdo es frágil y se filtra en la escritura que sirve para fijarlo. A esto contribuyen también los poemas que se encuentran a lo largo del libro y que refuerzan los momentos que forman la memoria.

Los lugares se acompañan con las personas, los padres, Betina -la hermana- y los abuelos, relacionados sobre todo con Argentina, los amigos, muy importantes en la época narrada, casi todos yugoslavos junto con las compañeras de la escuela secundaria en Trieste. Sin retórica y con eficacia narrativa, al lado de la importante figura del padre, Octavio, introducido con su nombre de pila, que atraviesa el relato con su fuerza intelectual y su sensatez para resolver situaciones de riesgo o precariedad doméstica, emerge la figura de la madre, Elvira Dolores, para todo el mundo Chiquita. La figura de la madre, desde un lugar menos expuesto en la narración, es a mi entender, insustituible en el desarrollo de la novela. Es ella quien da el impulso a la joven para 
vencer los sinsabores, la que la ayuda, alienta, protege y empuja -en definitiva- a crecer. Orgullosa de cierta ascendencia indígena, ella «Aceptó cada acontecimiento y trató de vivir feliz en él. Hizo suya la historia de mi padre. Lo vivido por él y con él le fue proprio. Y así nos lo transmitió» (55).

La metáfora pedalear / vivir conecta la escena con el título del libro y, fundamentalmente, relaciona los elementos esenciales de la ficción: sueño, imaginación, carencia, vida.

Otras mujeres son las abuelas, muy distintas entre sí pero igualmente importantes, la paterna María, istriana con pasaporte italiano por las circunstancias de la historia y Sara, la abuela materna, perteneciente a la familia española. Es un amplio universo que va de Europa a América, formado por lazos familiares que constituyen el pasado de la protagonista, cuyo recuerdo funda su personalidad.

Cruzando el río en bicicleta es la imagen que se le presenta a la abuela inmigrante que vive en una pequeña ciudad costera lejana de su tierra natal, del otro lado del mar, donde hay un río que hay que cruzar para ir al trabajo:

A María, lo que más le molestaba -cuenta la narradora- era que cada vez que tenía que bajar las escaleras para subir al bote, los hombres le miraran las bombachas. [...] Ese breve trayecto creaba en ella una incomprensible inquietud interior. A menudo soñaba que cruzaba el río en bicicleta flotando sobre el agua (68).

En realidad, la metáfora remite a las dificultades de la vida que marcan la existencia de estos -y de todos- los migrantes. Cruzar el río, podría pensarse además, como el deseo siempre insatisfecho que incita a la escritura diferida: es la nieta quien, definitivamente, cruza el océano, escribe la novela y ... pedalea.

\section{Argentina}

Los tres países que forman la historia de esta joven mujer aportan distintos valores que se mezclan y contribuyen a la adquisición de una educación internacional: la Yugoslavia de Tito con su sistema comunista que favorece el sentido de pertenencia; Argentina, el país que ofreció un futuro a millones de migrantes europeos, entre los cuales sus abuelos, y que medio siglo después es víctima de una dictadura que los expulsa y, para concluir, el futuro, representado por Trieste con sus muchas posibilidades. El pasado ocupa un espacio más amplio con respecto a un presente que se está viviendo. Como afirma la autora:

Traducir los fragmentos de Cruzando en los que existía una distancia histórica resultó ser más sencillo. Me refiero, por ejemplo, a los episodios referidos a la 
inmigración en Argentina a comienzos del siglo XX o a los de la primerísima infancia de la protagonista. En cambio, los episodios referidos a la historia reciente han requerido más de una adaptación, explicación, búsqueda del vocablo justo y, en algunos casos, del corte definitivo del fragmento (Prenz, 2017, en prensa).

Es evidente la importancia de la época existencial de referencia; la juventud que determina el punto de vista a través del cual el recuerdo queda marcado en la personalidad de la narradora con el entusiasmo y la vitalidad que caracterizan dicha edad.

La joven mujer cuenta de una familia muy querida, en donde sobresalen los abuelos, que son los que transmiten los recuerdos y la continuidad de la historia junto con los principios morales. Los abuelos paternos, emigrantes istrianos, viajaron a Argentina entre las dos guerras, durante el gobierno de Mussolini que italianizó a la fuerza la región, política que obligó a la emigración de los antifascistas. Son testigos de las atribuladas vicisitudes histórico-políticas que repetidamente han implicado dramáticamente en el siglo pasado las zonas de frontera nordoriental de Italia. La abuela María -a quien se alude en el título- es la inmigrante istriana con pasaporte italiano que se vuelve argentina, porque en Argentina, reflexiona la narradora, «Las lenguas, siempre las lenguas, murmuraban en la memoria de mi abuela. La de los eslavos: polacos, rusos, croatas, eslovenos; la de los italianos, del norte y del sur. Todas ellas se compenetraban en esa identidad tan argentina conformada de las idiosincrasias de cada uno» (48). Son precisamente los abuelos istrianos los que representan el vínculo con Argentina; los recuerdos de la niñez pasada en Ensenada están estrechamente relacionados con ellos:

Mis abuelos nos llevaban a pescar. El río estaba a pocos pasos. [...] Con mi hermana curioseábamos ese río y rastreábamos algunos colores que mi abuela mencionaba. [...] Mi abuelo se sentaba en un amarre y, siempre con su ritmo lento, tiraba el anzuelo en el agua. [...] Él fumaba. Nosotras jugábamos. Veo su espalda, su sombrero y ese río que no se acaba $(47,48)$.

Argentina es el país donde se llega para formar una nueva existencia gracias al duro trabajo de los abuelos, donde María «No se entristecía, marcaba el ritmo de su marcha de entonces: se levantaba, tomaba mate, planchaba, cocinaba, cruzaba el río con el bote, trabajaba en el frigorífico, volvía, comía, retomaba las tareas de la casa, [...]. Su marido había sido capataz y sus hijos habían estudiado» (49). Es el lugar también que expulsa a los padres, joven matrimonio con dos niñas, que se aleja para escapar de la violencia de la dictadura:

Llegamos a Belgrado dejando una Argentina atormentada. Junio de 1975. No sé si tan pequeña percibía la tragedia. Los recuerdos son pocos. Autos 
quemados por las esquinas de la ciudad de La Plata, hombres en torno a la Facultad de Humanidades con el arma apuntada, un auto Ford sin chapa frente a nuestra casa y, además, escritos por las paredes de la universidad que decían que mi padre era un hijo de puta (11).

Finalmente, en los últimos capítulos de la edición en castellano, la violencia de la historia de finales del siglo XX -en Argentina y en la región balcánica- es narrada con toda la emoción que provocó en las muchachas y muchachos de entonces.

\section{Yugoslavia}

La abuela María y el abuelo Grgo son los que representan el primer lazo entre Europa y América. Conocen poco Yugoslavia y por esto:

Cuando quería hablar con mis abuelos de Yugoslavia no me prestaban mucha atención. Por esas vicisitudes de la historia Belgrado se había convertido en la capital del país donde ellos habían nacido, pero sabían poco de ella. Me hablaban sólo de Istria, de sus caminos, de los pueblitos donde se hacían las fiestas, de sus canciones (46).

Es la abuela quien aparece con más insistencia en el recuerdo de la narradora. Ella era istriana, emigró a Argentina muy joven, separándose de la familia puesto que: «Mi abuela no volvió a ver nunca más a su madre, tampoco a su padre ni a algunos de sus hermanos y sabía que iba a ser así» (39). A pesar de que la memoria de la tierra natal vuelve a menudo en el relato de la anciana mujer:

Nos levantábamos con mis hermanos a las cinco de la mañana e íbamos a la escuela, pero por poco tiempo, después llegó la guerra, decía. Y no se avergonzaba de no saber escribir. [...] Contaba también que con los austríacos se vivía bien, había respeto. -Con los italianos cambió todo. A pesar de que en los documentos siguió siendo italiana, no conservó jamás un lindo recuerdo de ellos. Hablaba de su pasado con orgullo, a veces con cierta soberbia, pero dejaba siempre una sensación de ternura. [...] Mis abuelos recordaban los tiempos del Imperio austro-húngaro y más tarde de las prepotencias de los fascistas. $(40,46)$

Yugoslavia es el país donde la joven protagonista transcurre su juventud: «Siempre pensé que ese fue el momento más feliz de mi vida. La identificación total con lo que me circundaba» (6). La familia se traslada a Belgrado donde queda cuatro años, sin perder nunca el contacto con su realidad después del cambio de residencia a Trieste. El encuentro con la capital serbia es muy fuerte: «Botas, humo, mucho humo, olores agradables y desagradables, cigarrillo - porque los serbios fuman mucho- y el perfume 
a grapa que emana de la boca y de los poros de la gente. [...] Todo me atraía, fascinaba, conducía a tiempos muy lejanos, a una forma primigenia de la humanidad donde ternura, ingenuidad, buenos sentimientos y brutalidad se confundían» $(6,7)$.

Esta tierra es muy importante en la formación sentimental de la joven mujer, hasta llegar al penúltimo capítulo, titulado «Ex», donde se destruye el referente para transformarlo en personaje con el cual el yo de la narradora conversa:

A veces dialogo con Yugoslavia. Es simpática. [...] Me dice que todas las cosas acaban en algún momento. Cuando muere un ser querido, muere una parte de nosotros. [...] -Eras Absurda. ¡Cuántas cosas ilógicas te sucedían! Nos divertíamos. Nos queríamos. Creíamos. [...] Y yo gozaba de tus olores, de tus certezas. [...] A veces lloro. Lágrimas contenidas. Plenas. Yugoslavia sonríe. Toma una botella. De aguardiente, naturalmente. Sirve, lenta, en los vasitos. Me mira a los ojos, brindamos. Se va. (96)

Yugoslavia se vuelve categoría antropológica, se «humaniza» llegando a ser la amiga con quien compartir pensamientos y emociones. De forma especial se percibe el dolor de ya no poder sentirse parte de aquella tierra, porque «Yo quería seguir siendo yugoslava» (91).

El libro acaba con una referencia a Laura Papo Bohoreta (1891-1942), -tema de investigación heredado del padre- autora de poemas, cuentos, ensayos y obras teatrales, única dramaturga sefardí de Bosnia que vivió en Sarajevo, ciudad que, de alguna forma, representa una unión perdida, puesto que «Es un microcosmos, centro del mundo que, como todo centro según la enseñanza de los esotéricos, contiene todo el mundo» (98).

Red de seguridad en el momento de la huida de la tragedia de los Balcanes, es Trieste, ciudad donde la familia se muda justo un poco antes de la muerte de Tito. El traslado a Italia se realiza en 1979 y es un nuevo viaje de emigrantes:

Nosotras íbamos sentadas atrás; las valijas, las cajas, los libros no entraban en el baúl, tampoco las lámparas de las que mi madre no quería despegarse. El abat jour era bordó, bien grande. Una lámpara de pie más grande que yo. Me la pusieron de sombrero y así viajé las diez horas. No nos preocupaba mucho el estilo balcánico. No le dábamos peso a las formas. (60)

Trieste representa un pasado reciente que continúa en el presente, un lugar desde donde se descubre la extraordinaria belleza de Italia y que, de alguna forma, ocupa un lugar especial, ya que pertenece a la experiencia actual de la narradora. La parte relativa a la narración de la ciudad italiana, de hecho, es en presente: "Qué ciudad encantadora y qué gente encantadora. Se vive como suspendidos en el aire, fuera de 
cualquier dimensión de lo real. Concentrados en sí mismos. [...] Vuelvo, siempre vuelvo. Porque perfección y ausencia provocan goce y percepción de centralidad» (61).

Como señala Silvana Serafin, en los relatos de migración: «Lo spazio si configura come luogo per riconquistare un'identità composita, per qualificare ad un tempo l'appartenenza [americana e l'eredità italiana], nonostante la sua identificazione di nuovo 'esilio'» (Serafin, 2014, 15). Por esto los tres lugares en donde se desarrolla la historia son importantes para definir la personalidad de la narradora. Cada uno de ellos con sus colores, olores, remite a un pasado de alegría y de dolor y deja vislumbrar posibilidades futuras, cargándose de un significado simbólico.

Los tres países, además, contribuyen de forma distinta en la formación internacional de la protagonista. Junto con la Yugoslavia de Tito con su ideología que acompaña su primera juventud en la inclusión del mundo que la rodea, continúa fuerte el lazo con Argentina, la tierra que ha acogido millones de migrantes, entre los cuales también a la familia paterna de la narradora y que, a pesar del exilio al que obligó los padres de la joven, continúa perteneciendo al presente de su historia. Finalmente, Trieste, la ciudad en donde se forma intelectualmente.

\section{Los finales}

En un primer momento, el libro se escribe en español, lengua materna de la narradora, en un segundo momento se realiza una autotraducción al italiano y, además, se anuncia otra autotraducción en serbocroata. Puesto que en la traducción italiana hay unos cambios - un final distinto-, prefiero hablar de versión más que de traducción. Esto se justifica también por el hecho que cada re-escritura implica un acercamiento distinto con respeto al original hasta transformarlo en otro texto. De este tema Ana Cecilia Prenz Kopušar ha comentado en su artículo «Reflexiones sobre la autotraducción desde la mirada del autor» (2017, en prensa), donde declara:

Por primera vez, en la redacción de un texto, me encuentro en la particular condición de cumplir tres roles: el de autora de un libro, el de estudiosa entre otras cosas, también de la traducción- y el de autotraductora; en mi caso, autotraductora a dos lenguas: al italiano y al serbio partiendo del original escrito en español. [...] Determinadas circunstancias de la vida me han llevado a hablar tres lenguas: el español en su variante argentina, el italiano y el serbocroata. Con respecto al español y al serbocroata, desde la primera infancia fui adquiriendo ambas lenguas de manera casi simultánea, la primera, en el entorno estrictamente familiar [...], la segunda, en la guardería y en el contexto de la ciudad. Esto por haber nacido en Yugoslavia de padres argentinos. A los cuatro años volví a Argentina y perdí el uso cotidiano de 
la lengua eslava. Esta última se mantuvo viva solo a través de canciones o intercambiando algún vocablo o expresión con los abuelos paternos. La recuperación del serbocroata o, diría, su nuevo aprendizaje, se dio a los diez años, una vez que volví a vivir a Belgrado. En algún lugar del inconsciente esta lengua se mantuvo latente, dado que su recuperación fue rápida, teniendo también en cuenta la edad: once años. Después de un mes y medio de escuela ya comunicaba con los compañeros en serbio ${ }^{1}$. El italiano, en cambio, lo aprendí en edad adolescente (a los quince) en Trieste, donde comencé mis estudios en la escuela secundaria. El aprendizaje, en este caso, fue sistemático y a través del estudio; se dio de manera menos espontánea, no obstante, tuviera una fluida comunicación cotidiana con el entorno no familiar. Puedo afirmar que percibo mi relación con las lenguas, así como se fue dando su mismo proceso de aprendizaje. Una es la lengua familiar (íntima y de la escritura), la otra del juego, la tercera del estudio. Esta simplificación, pero que en realidad no es tal, define también mi actitud en el momento de enfrentar el trabajo de autotraducción. (Prenz, 2017, en prensa)

En la edición italiana se cambia el final y se añade un 'Epílogo', representando así casi otro libro. Según lo que declaró la autora, la versión italiana, posterior al original, viene a ser más reflexiva, menos 'necesaria' y urgente que la primera, en castellano ${ }^{2}$. En su conclusión resulta evidente la declaración de amor hacia los lugares que han marcado la existencia de la protagonista:

Io ho tre amori non li enumero sono scontati. Con il primo mi sono formata, ho costruito i miei principi, sono diventata persona. Ho condiviso. Condiviso. Il secondo è un amore scapigliato, e per quanto crudele, vissuto con allegria. Con lui ho capito gli estremi, la leggerezza, l'umorismo, ridere, sempre pronti ma ridere, ma anche il terrore, la violenza, la solitudine. Il terzo mi ha fatto godere della bellezza, unica e assoluta. E stato il più doloroso perché sofisticato, complesso a volte contorto. Lontano dalla mia natura semplice. Con la sua arte mi ha ricordato giorno dopo giorno, la grandezza umana (118).

Sin embargo, lo que sobresale es su ser latinoamericana, como ella misma subraya: «Sono fra le montagne e da questo luogo, forse ancora come un tempo, vivo il mio essere latinoamericana» (118). Declaración que la reconcilia con sus muchas identidades y le permite poder asumirlas todas. Argentina, de hecho, es el lugar de nacimiento de sus padres, es el país que recibió a sus abuelos emigrantes y millones de extranjeros en busca de un futuro, ofreciéndoles la posibilidad de formar una familia y de transmitir

1 Cuando aludo al serbio me estoy refiriendo a la variante ekavski y oriental de la lengua serbocroata.

2 Declaración que la autora hizo durante la presentación de su libro en Venecia, el 28 de noviembre, en el Cultural Flow Zone (CFZ) de la Universidad Ca’ Foscari de Venecia. 
su experiencia a las generaciones futuras. Es el país que más ha conocido la relación con el otro en el respeto y la inclusión y que continúa siendo muy importante para la protagonista, adonde viaja muy a menudo.

Es un libro sugestivo que, a través de la escritura de una narración de formación, abarca dos continentes y parte de la Historia del siglo XX; las grandes migraciones de Europa a las Américas, los exilios debidos a las dictaduras del Cono Sur de la segunda parte del 900 y finalmente la terrible guerra que marcó la disolución de la Yugoslavia y el genocidio étnico.

\section{Bibliografía}

Boria, A., Prenz A. C., I miei tre mondi dentro un diario, Il Piccolo, 21. 4. 2015, http:// ilpiccolo.gelocal.it/tempo-libero/2015/04/21/news/ana-cecilia-prenz-i-miei-tremondi-dentro-un-diario-1.11281548?refresh_ce [15. 1.2017].

Crolla, A., Migración, autoficción y autotraducción en: Cruzando el río en bicicleta de Ana Cecilia Prenz Kopušar, Oltreoceno 13, 2017, pp. 197-207.

Lejeune, P., Le Pacte autobiographique, Paris 1975.

Prenz Kopušar, A. C., Cruzando el río en bicicleta, Buenos Aires 2015.

Prenz Kopušar, A. C., Attraversando il fiume in bicicletta, Trieste 2016.

Prenz Kopušar, A. C., Crolla, A., Entrevista on line (diciembre 2016. Inédito).

Prenz Kopušar, A. C., Reflexiones sobre la autotraducción desde la mirada del autor, Hilo de la Fábula, en prensa, 2017.

Puertas M., Francisco E., Una puesta al día de la teoría autoficticia como contrato de lectura autobiográfica, Sigma: Revista de la Asociación Española de Semióta 14, 2005, pp. 299-330.

Scarano, L., Palabras en el cuerpo: literatura y experiencia, Buenos Aires 2007.

Serafin, S., Letteratura migrante. Alcune considerazioni per la definizione di un genere letterario, Altre Modernità, giugno 2014, pp. 1-17, riviste.unimi/index.php/ AMonline/article/riew/4117 [25. 10. 2017]. 
Susanna Regazzoni

\section{Med Argentino in Italijo prek Jugoslavije: Cruzando el rio en bicicleta Ane Cecilie Prenz Kopušar}

Ključne besede: Argentina, Italija, Jugoslavija, avtofikcija, spomin, identiteta

Besedilo Cruzando el río en bicicleta žanrsko spada v avtofikcijo: gre namreč za zgodbo, ki se nahaja na polovici poti med pripovedništvom in biografijo in ki predstavi spomine iz otroštva in mladosti neke ženske, ki se odvijajo med Beogradom, Buenos Airesom in Trstom. Svoje osebne izkušnje doživlja skozi tri države in tri različne jezike v letih, ki segajo od argentinske vojaške diktature do tragičnih vojn v bivši Jugoslaviji. Gre za razvojni roman, ki se ukvarja $\mathrm{z}$ identiteto Argentine, države, osnovane na prispevku evropskih priseljencev od konca 19. pa do sredine 20. stoletja - v tem primeru gre za avtoričino istrsko družino - in zaznamovane z izkušnjo izgnanstva veliko mladih, ki so med diktaturo konec 20. stoletja odpotovali v tujino. Delo, ki je bilo v prvi izdaji objavljeno $\mathrm{v}$ španščini, je avtorica sama prevedla v italijanščino in $\mathrm{mu}$ $\mathrm{v}$ prevodu namenila drugačen konec. 
Susana Regazzoni

\section{Between Argentina and Italy, passing through Yugoslavia: Crossing the River by Bicycle by Ana Cecilia Prenz Kopušar}

Keywords: Argentina, Italy, Yugoslavia, self-fiction, memory, identity

Crossing the River by Bicycle (Cruzando el río en bicicleta) is a text that can be defined as self-fiction, that is, a story that is halfway between narrative and biography and that presents the memories of a woman's childhood and adolescence that take place between Belgrade, Buenos Aires and Trieste. Her experiences thus occur in three countries and three different languages over a timeframe years that goes from the Argentine military dictatorship to the tragedy of the wars in the former Yugoslavia. It is a novel that refers to the identity of Argentina, a country constituted by the contribution of European immigrants from the late nineteenth century to the midtwentieth century - in this case the Istrian family of the author - and marked by the exile of the many young people who travelled abroad during the dictatorships of the second half of the twentieth century. The first edition was published in Spanish, and has been translated into Italian by the same author with a different ending. 